\title{
UNIQUENESS OF INVARIANT MEANS FOR MEASURE-PRESERVING TRANSFORMATIONS
}

BY

\author{
JOSEPH ROSENBLATT
}

\begin{abstract}
For some compact abelian groups $X$ (e.g. $T^{n}, n>2$, and $\Pi_{n=1}^{\infty} Z_{2}$ ), the group $G$ of topological automorphisms of $X$ has the Haar integral as the unique $G$-invariant mean on $L_{\infty}\left(X, \lambda_{X}\right)$. This gives a new characterization of Lebesgue measure on the bounded Lebesgue measurable subsets $\beta$ of $R^{n}, n>3$; it is the unique normalized positive finitely-additive measure on $\beta$ which is invariant under isometries and the transformation of $R^{n}:\left(x_{1}, \ldots, x_{n}\right) \mapsto\left(x_{1}+x_{2}, x_{2}, \ldots, x_{n}\right)$. Other examples of, as well as necessary and sufficient conditions for, the uniqueness of a mean on $L_{\infty}(X, \beta, p)$, which is invariant by some group of measure-preserving transformations of the probability space $(X, \beta, p)$, are described.
\end{abstract}

0. Introduction. Let $\beta$ be the ring of bounded Lebesgue measurable sets in $R^{n}$ or in $S^{n}$, the $n$-dimensional unit sphere in $R^{n+1}$, and let $\lambda_{n}$ be the Lebesgue measure on $\beta$ normalized by $\lambda_{n}\left(J^{n}\right)=1$, where $J=[0,1]$, or by $\lambda_{n}\left(S^{n}\right)=1$ respectively. The classical characterization by Lebesgue of $\lambda_{n}$ is that it is the unique positive real-valued function $f$ on $\beta$ which satisfies these three conditions.

(a) $f\left(J^{n}\right)=1$ or $f\left(S^{n}\right)=1$ respectively,

(b) $f$ is invariant under isometries,

(c) $f$ is countably-additive.

In 1923, Banach [1] studied the question of Ruziewicz as to whether $f$ is still unique when (c) is replaced by

$\left(\mathrm{c}_{0}\right) f$ is finitely-additive.

Banach gave a negative answer to this question for $R^{1}, R^{2}$, and $S^{1}$; but for $R^{n}$, $n \geqslant 3$, or $S^{n}, n \geqslant 2$, this question is still unanswered.

In this paper we will prove a theorem which comes close to a solution of this problem for $R^{n}, n \geqslant 3$.

THEOREM 3.10. If $f$ satisfies (a), (b), and $\left(\mathrm{c}_{0}\right)$, and if $f$ is invariant under the transformation of $R^{n}, n \geqslant 3$, given by $\left(x_{1}, x_{2}, \ldots, x_{n}\right) \mapsto\left(x_{1}+x_{2}, x_{2}, \ldots, x_{n}\right)$, then $f=\lambda_{n}$.

Our method of proof will give several other theorems similar to this one. In Theorem 3.10, we do not need the full strength of (b); we need only know that $f$ is absolutely continuous with respect to $\lambda_{n}$ i.e. for $N \in \beta, f(N)=0$ whenever $\lambda_{n}(N)=0$. The fact that for $R^{n}, n \geqslant 3$, or $S^{n}, n \geqslant 2$, (a), (b), and (c $\left.c_{0}\right)$ imply this absolute continuity of $f$ is an observation of Tarski which is proved like this.

Received by the editors March 3, 1980; presented to the Society, January 10, 1981.

AMS (MOS) subject classifications (1970). Primary 28A70, 43A07, 43A40. 
Because there are paradoxical decompositions by isometries of $J^{n}, n>3$, or $S^{n}$, $n \geqslant 2$, for $N \in \beta$ when $\lambda_{n}(N)=0, N$ can be packed by finite decomposition and isometries into open sets of arbitrarily small diameter; hence $f(N)=0$ if $\lambda_{n}(N)=0$ with $N \in \beta$. This implication is not true for $R^{1}, R^{2}$, or $S^{1}$; for example, see [8]. Therefore, we need to assume absolute continuity instead of (b) in proving this analogue of Theorem 3.10 which holds for all $n \geqslant 2$.

THEOREM 3.11. If $f$ satisfies (a) and $\left(\mathrm{c}_{0}\right)$, and $f$ is absolutely continuous with respect to $\lambda_{n}, n \geqslant 2$, then $f=\lambda_{n}$ as long as $f$ is invariant under these three transformations of $R^{n}, n \geqslant 2$.

$$
\begin{aligned}
& \left(x_{1}, x_{2}, \ldots, x_{n}\right) \mapsto\left(x_{1}+1, x_{2}, \ldots, x_{n}\right), \\
& \left(x_{1}, x_{2}, \ldots, x_{n}\right) \mapsto\left(x_{1}, x_{1}+x_{2}, \ldots, x_{n}\right), \\
& \left(x_{1}, x_{2}, \ldots, x_{n}\right) \mapsto\left(x_{2}, \ldots, x_{n},(-1)^{n+1} x_{1}\right) .
\end{aligned}
$$

These results can also be viewed as proving the existence of a new kind of paradoxical decomposition of the cube $J^{n}, n>2$. Already in [11], it was shown that for $J^{n}, n>3$, there exist paradoxical decompositions by isometries with Borel parts modulo some isometry-invariant ideal of Borel sets. Now, by Tarski's equivalence of the existence of paradoxical decompositions and the nonexistence of invariant finitely-additive measures (see [9] for example), our main theorems prove that, for any invariant ideal in $\beta$ which contains a set of positive measure and also contains all null sets, there exist paradoxical decompositions of $J^{n}, n \geqslant 2$, modulo this ideal. But here the transformations being used come from the group generated by the three transformations of Theorem 3.11 and not just from the group of isometries of $R^{n}$ demanded in the original problem of Ruziewicz. When using just isometries for the transformations, the existence of paradoxical decomposition, modulo an ideal as before, is still unknown.

The techniques of functional and harmonic analysis used to prove the main theorems are perhaps best understood when one considers invariant linear functionals instead of invariant finitely-additive measures. For instance, the problem of Ruziewicz for $R^{n+1}, n \geqslant 1$, has this alternate formulation. Let $L_{\infty}\left(S^{n}\right)$ denote the bounded Lebesgue measurable functions on the $n$-sphere in $R^{n+1}$. The Lebesgue measure $\lambda_{n}$ determines a linear functional $I_{n}$ on $L_{\infty}\left(S^{n}\right)$ given by $I_{n}(f)=\int f d \lambda_{n}$ for all $f \in L_{\infty}\left(S^{n}\right)$. This linear functional $I_{n}$ has the properties (1) $I_{n}(1)=1$, (2) $I_{n}(f) \geqslant 0$ if $f \geqslant 0$, and (3) $I_{n}\left({ }_{g} f\right)=I_{n}(f)$ if $f \in L_{\infty}\left(S^{n}\right)$ and $g$ is an isometry of $S^{n}$. A linear functional with properties (1)-(3) is an invariant mean of $L_{\infty}\left(S^{n}\right)$, invariance being with respect to the group of isometries of $S^{n}$. The problem is this: is $I_{n}$ the unique isometry-invariant mean on $S^{n}$ ?

The notion of an amenable group and the importance of amenability in harmonic analysis has been recognized by many authors. See Greenleaf [4] for a survey of amenability. In this context, we have the modern version of the problem that Banach studied. Let $G$ be an infinite compact group and let $\lambda_{G}$ be the Haar measure on $G$ normalized by $\lambda_{G}(G)=1$. Let $L_{\infty}(G)$ denote the usual equivalence classes of $\lambda_{G}$-measurable essentially bounded functions on $G$. The measure $\lambda_{G}$ 
determines a mean $I_{G}$ on $L_{\infty}(G)$ by $I_{G}(f)=\int f d \lambda_{G}$ for all $f \in L_{\infty}(G)$. This mean is invariant under left (or right) translations and group inversion in $G$. The problem is this: is $I_{G}$ the unique mean on $L_{\infty}(G)$ which is invariant under left-translations? Independently, Granirer [3] and Rudin [13] showed that if $G$ is amenable as a discrete group, then there are other invariant means on $L_{\infty}(G)$; Rosenblatt [12] showed that there are even $2^{c}$ mutually singular invariant means on $L_{\infty}(G)$ if $G$ is amenable as a discrete group. The techniques used in [12] show that for the classical problem on $L_{\infty}\left(S^{1}\right)$ studied by Banach, there are also $2^{c}$ mutually singular invariant means. Moreover, in Rosenblatt [12] it is shown that the existence of many invariant means on $L_{\infty}(G)$ can imply the amenability of $G$ as a discrete group. See [12] for the details.

However, it is still unsolved whether there can be any infinite compact group $G$ such that $I_{G}$ is the unique invariant mean on $L_{\infty}(G)$. Worse yet, until recently the following slightly more general problem was not resolved. If $G$ is a semigroup of measure-preserving transformations of a probability space $(X, \beta, p)$, does there exist more than one $G$-invariant mean on $L_{\infty}(X, \beta, p)$ ? Of course, the $G$-invariant mean $p$ determines one $G$-invariant mean $I$ on $L_{\infty}(X, \beta, p)$ by $I(f)=\int f d p$ for all $f \in L_{\infty}(X, \beta, p)$. The question is whether $I$ is the only invariant mean. In del Junco and Rosenblatt [2], it is shown that when $G$ is a countable amenable semigroup, and $(X, \beta, p)$ is nonatomic, there are other $G$-invariant means. This is an abstract analogue for countable semigroups of [3] and [13]. Also, it is easy to see that if $G$ is too large (e.g. consisting of all measure-preserving transformations of $(X, \beta, p)$ ), then there is a unique invariant mean. What is important is a case like the prior ones where $G$ is a group of natural geometrical transformations of some probability space, and, moreover, $G$ is not a large group but is countable or even finitely generated.

In $\S 1$, this more general problem is studied and necessary and sufficient conditions for the uniqueness of $I$ are given. In $\$ 3$, an application of these theorems shows that the topological automorphisms of the torus $T^{n}, n>2$, form a group with two generators acting on $T^{n}$ as measure-preserving transformations such that the Haar integral is the unique invariant mean for $L_{\infty}\left(T^{n}, \beta, \lambda_{T^{n}}\right)$. Both Theorems 3.10 and 3.11 are consequences of this result.

When this paper was essentially finished, we received a preprint [5] from V. Losert and H. Rindler where some theorems similar to the theorems in $\$ 3$ were obtained. They use [2], but apparently were unaware of [14] which inspired us. Although obtained independently of [5], both Proposition 3.5 and Theorem 3.6 have been written here to show more clearly their similarity to the main theorem in [5] as well as their dependence on $\S 1$. Also, a debt is owed to J. Mycielski for his many helpful suggestions about the contents of this paper.

1. Asymptotically invariant nets. Let $(X, \beta, p)$ be a probability space and let $G$ be a group of measure-preserving transformations of $(X, \beta, p)$. For a $\beta$-measurable function $f: X \rightarrow \mathbf{C}$ into the complex numbers $\mathbf{C}$ and a mapping $g \in G,{ }_{g} f(x)=$ $f(g(x))$ define another measurable function ${ }_{g} f: X \rightarrow C$. In this way, $G$ acts as a 
family of bounded linear operators on $L_{\infty}(X, \beta, p)$, the linear space of $p$-equivalence classes of $p$-essentially bounded measurable functions. A $G$-invariant mean is a linear functional $\varphi \in L_{\infty}^{*}(X, \beta, p)$ which is a mean (i.e. $\varphi(f) \geqslant 0$ whenever $f \geqslant 0$ and $\varphi(1)=1)$ and which satisfies $\varphi\left({ }_{g} f\right)=\varphi(f)$ for all $g \in G$ and $f \in L_{\infty}(X, \beta, p)$. Because $p$ is $G$-invariant, $I(f)=\int f d p$ defines a $G$-invariant mean on $L_{\infty}(X, \beta, p)$.

Suppose that there is a measurable set $A$ with $0<p(A)<1$ and $p\left(g^{-1}(A) \Delta A\right)=$ 0 for all $g \in G$. Then $I$ is trivially not unique because $I_{A}(f)=\left(\int_{A} f d p\right) / p(A)$ defines another $G$-invariant mean on $L_{\infty}(X, \beta, p)$. So we will assume throughout this section that $G$ is ergodic; no such $A$ exists.

The existence of $G$-invariant means besides $I$ can be characterized by a type of Følner condition. First, let $\varphi$ be any $G$-invariant mean on $L_{\infty}(X, \beta, p)$ with $\varphi \neq I$. Then define $m(E)=\inf \left\{\sum_{i=1}^{\infty} \varphi\left(1_{E_{i}}\right):\left(E_{i}\right)\right.$ is a measurable partition of $\left.E\right\}$ for each measurable set $E$. The set function $m$ is a $G$-invariant countably-additive measure on $(X, \beta)$. Moreover, because $1_{E}=0$ in $L_{\infty}(X, \beta, p)$ if $p(E)=0, m$ is absolutely continuous with respect to $p$. This is the main point at which it is critical that our linear space $L_{\infty}(X, \beta, p)$ consists of equivalence classes of functions. Some discussion of this point and what happens if our linear space is $\mathcal{L}_{\infty}(X, \beta, p)$, all essentially bounded measurable functions not identified in $p$-equivalence classes, will be given at the end of $\S 3$. But here this absolute continuity forces $m=c p$ where $c$ is some constant, $0 \leqslant c \leqslant 1$. Because $\varphi \neq I, c \neq 1$. Let $\varphi_{0}=(\varphi-c I) /(1-c)$. Then $\varphi=$ $c I+(1-c) \varphi_{0}$ and $\varphi_{0}$ is another $G$-invariant mean. We call this the canonical decomposition of the $G$-invariant mean $\varphi$.

Proposition 1.1. If there exists a G-invariant mean $\varphi$ with $\varphi \neq I$, then there exist a G-invariant mean $\varphi_{0}$ and an increasing sequence $\left(E_{n}\right)$ of measurable sets with $\lim _{n \rightarrow \infty} p\left(E_{n}\right)=1$, and $\varphi_{0}\left(1_{E_{n}}\right)=0$ for all $n \geqslant 1$.

Proof. Let $\varphi=c I+(1-c) \varphi_{0}$ be the canonical decomposition of $\varphi$. Fix a measurable set $E_{0}$ with $p\left(E_{0}\right)>0$. There is some measurable sets $E \subset E_{0}$ with $p(E)>0$ and $\varphi_{0}\left(1_{E}\right)=0$. Otherwise, for all measurable sets $E \subset E_{0}, p(E)=0$ if and only if $\varphi_{0}\left(1_{E}\right)=0$. We could then define $m_{0}$ in terms of $\varphi_{0}$ as $m$ was defined in terms of $\varphi$; one can show that at least for measurable sets $E \subset E_{0}, m_{0}(E)=0$ if and only if $p(E)=0$. In particular, $m_{0}\left(E_{0}\right) \neq 0$. But $m_{0}$ is a countably-additive measure with $(1-c) m_{0} \leqslant(1-c) \varphi_{0} \leqslant \varphi_{0}$. This means $(1-c) m_{0}=0$ and $m_{0}=0$ because any countably-additive positive measure $v$ on $(X, \beta)$ with $v(E)<\varphi_{0}\left(1_{E}\right)$, for all measurable sets $E$, must be identically zero. This is a contradiction.

Now use measure-theoretic transfinite induction to show that there exists a sequence $\left(D_{n}\right)$ of pairwise-disjoint measurable sets with $p\left(D_{n}\right)>0$ and $\varphi_{0}\left(1_{D_{n}}\right)=0$ for all $n \geqslant 1$, and with $\sum_{n=1}^{\infty} p\left(D_{n}\right)=1$. Let $E_{n}=\cup_{i=1}^{n} D_{i}$ for $n>1$ to get the desired sequence of measurable sets.

We use this proposition to get the next one. The set $\mathcal{P}$ will denote $\{f \in$ $L_{1}(X, \beta, p): f \geqslant 0$ and $\left.\int f d p=1\right\}$.

Proposition 1.2. If there exists a $G$-invariant mean $\varphi \neq I$, then there exist an increasing sequence $\left(E_{n}\right)$ of measurable sets with $\lim _{n \rightarrow \infty} p\left(E_{n}\right)=1$ and $a$ net $\left(f_{\alpha}\right) \subset \mathcal{P}$ such that $\lim _{\alpha}\left\|_{g} f_{\alpha}-f_{\alpha}\right\|_{1}=0$ for all $g \in G$ and, for all $n \geqslant 1$, there exists $\alpha_{0}$ such that for all $\alpha \geqslant \alpha_{0}, f_{\alpha}=0$ a.e. on $E_{n}$. 
Proof. Fix a $G$-invariant mean $\varphi_{0}$ and a sequence $\left(E_{n}\right)$ as in Proposition 1.1. Because $\mathcal{P}$ is $\omega^{*}$-dense in the positive part of the unit ball of $L_{\infty}^{*}(X, \beta, p)$, there exists a net $\left(f_{\alpha}\right) \subset \mathscr{\rho}$ with $f_{\alpha} \rightarrow \varphi_{0}$ pointwise on $L_{\infty}(X, \beta, p)$ i.e. $\lim _{\alpha} \int f_{\alpha} F d p=$ $\varphi_{0}(F)$ for all $F \in L_{\infty}(X, \beta, p)$. But $\varphi_{0}\left(1_{E_{n}}\right)=0$ for all $n \geqslant 1$; hence, $\lim _{\alpha} \int f_{\alpha} 1_{X \backslash E_{n}} d p=1$ for all $n \geqslant 1$. Therefore, by restricting $f_{\alpha}$ to $X \backslash E_{n}$ and normalizing the restriction, one can obtain a net, also denoted by $\left(f_{\alpha}\right)$, with $\left(f_{\alpha}\right) \subset \mathscr{P}$ such that $f_{\alpha} \rightarrow \varphi_{0}$ pointwise on $L_{\infty}(X, \beta, p)$ and, in addition, eventually $f_{\alpha}=0$ a.e. on any fixed $E_{n}$. Assume we have chosen such a net $\left(f_{\alpha}\right) \subset \mathcal{P}$. Using the method of Theorem 2.2 in Namioka [10] as applied to the group $G$ acting on $L_{1}(X, \beta, p)$, one concludes that some net $\left(h_{\alpha}\right)$ of convex combinations of $\left\{f_{\alpha}\right\}$ has the property that $\lim _{\alpha}\left\|_{g} h_{\alpha}-h_{\alpha}\right\|_{1}=0$ for all $g \in G$; of course, the net $\left(h_{\alpha}\right) \subset \mathcal{P}$ again. By starting with subnets of the original net $\left(f_{\alpha}\right)$, one can see easily from this that there exists a net $\left(f_{\alpha}\right) \subset \mathcal{P}$ such that $\lim _{\alpha}\left\|_{g} f_{\alpha}-f_{\alpha}\right\|_{1}=0$ for all $g \in G$ and such that, for any $n \geqslant 1, f_{\alpha}=0$ a.e. on $E_{n}$ eventually.

The next theorem will be proved in a way similar to the proof of [2, Theorem 2.4]. This idea was inspired by Namioka [10].

TheOREM 1.3. Assume that there exists a G-invariant mean $\varphi$ with $\varphi \neq I$. Then there exists an increasing sequence $\left(E_{n}\right)$ of measurable sets with $\lim _{n \rightarrow \infty} p\left(E_{n}\right)=1$, and there exists a net $\left(A_{\alpha}\right)$ of measurable sets with $p\left(A_{\alpha}\right)>0$ for all $\alpha$, such that

(1) for all $n \geqslant 1$, eventually $A_{\alpha} \subset X \backslash E_{n}$,

(2) for all $g \in G, \lim p\left(g^{-1}\left(A_{\alpha}\right) \Delta A_{\alpha}\right) / p\left(A_{\alpha}\right)=0$.

Proof. Let $\left(f_{\alpha}\right)$ be a net as in Proposition 1.2. Fix some $g_{1}, \ldots, g_{k} \in G$. Let $\theta_{\alpha}$ be the ordinate set of $f_{\alpha}$ in $X \times R^{+}$, that is $\theta_{\alpha}=\left\{(x, t): 0<t<f_{\alpha}(x)\right\}$, and let $\theta_{\alpha i}$ be the ordinate set of $f_{g_{\alpha}}$. Writing $p \times \lambda$ for the product of $p$ with Lebesgue measure $\lambda$ on $R^{+}$, we have by Fubini's Theorem, $p \times \lambda\left(\vartheta_{\alpha i}\right)=p \times \lambda\left(\vartheta_{\alpha}\right)=\left\|f_{\alpha}\right\|_{1}$ $=1$ and $p \times \lambda\left(\theta_{\alpha i} \Delta \theta\right)=\left\|_{g_{i}} f_{\alpha}-f_{\alpha}\right\|_{1}$. For any $\varepsilon>0$, if $\alpha$ is large enough, then we have

$$
p \times \lambda\left(\vartheta_{\alpha} \cap \bigcap_{i=1}^{k} \vartheta_{\alpha i}\right)>(1-\varepsilon / 2) .
$$

Now recall that for a measurable set $U \subset X \times R^{+}, p \times \lambda(U)=\int_{0}^{\infty} p\left(U_{t}\right) d t$ where $U_{t}=\{x:(t, x) \in U\}$. Also note that

$$
\left(\vartheta_{\alpha} \cap \bigcap_{i=1}^{k} \Theta_{\alpha i}\right)_{t}=\left\{t_{\alpha} \geqslant t\right\} \cap \bigcap_{i=1}^{k}\left\{f_{\alpha} \circ g_{i} \geqslant t\right\} \text {. }
$$

Thus, (1) may be written as

$$
\int_{0}^{\infty} p\left(\left\{f_{\alpha} \geqslant t\right\} \cap \bigcap_{i=1}^{k}\left\{f_{\alpha} \circ g_{i} \geqslant t\right\}\right) d t>(1-\varepsilon / 2) \int_{0}^{\infty} p\left(\left\{f_{\alpha}>t\right\}\right) d t .
$$

Therefore, by (2), there must be a set of $t$ of positive Lebesgue measure, and hence at least one $t>0$, such that

$$
p\left(\left\{f_{\alpha} \geqslant t\right\} \cap \bigcap_{i=1}^{k}\left\{f_{\alpha} \circ g_{i} \geqslant t\right\}\right)>(1-\varepsilon / 2) p\left(\left\{f_{\alpha} \geqslant t\right\}\right) .
$$


Hence, there exists some $t$ such that for all $i=1, \ldots, k$, we have

$$
p\left(\left\{f_{\alpha} \geqslant t\right\} \cap g_{i}^{-1}\left(\left\{f_{\alpha} \geqslant t\right\}\right)\right)>(1-\varepsilon / 2) p(\{f \geqslant t\}) .
$$

Since $\left\{f_{\alpha}>0\right\} \subset X \backslash E_{n}$ eventually, we can take the set $\left\{f_{\alpha} \geqslant t\right\}$ in (4) to be contained in $X \backslash E_{n}$. Hence, for all finite $F \subset G, \varepsilon>0$, and $n>1$, there exists a measurable $A, p(A)>0$, such that $A \subset X \backslash E_{n}$, and for all $g \in F$, $p\left(g^{-1}(A) \Delta A\right) / p(A)<\varepsilon$. By a suitable indexing, we get a net $\left(A_{\alpha}\right)$ as stated in the theorem.

A net of sets with property (2) of Theorem 1.3 will be called asymptotically invariant. The conditions on $\left(E_{n}\right)$ and (1) above force $\lim _{\alpha} p\left(A_{\alpha}\right)=0$. If this is the case, as well as (2), then $\left(A_{\alpha}\right)$ is an arbitrarily small asymptotically invariant net. If $\left(A_{\alpha}\right)$ is asymptotically invariant and $\left(p\left(A_{\alpha}\right)\right)$ is bounded away from 1 , then $\left(A_{\alpha}\right)$ is called a nontrivial asymptotically invariant net. Given a net as in Proposition 1.2, any $\omega^{*}$-limit of $\left(1_{A_{\alpha}} / p\left(A_{\alpha}\right)\right)$ in $L_{\infty}^{*}(X, \beta, p)$ will be a $G$-invariant mean on $L_{\infty}(X, \beta, p)$ satisfying the properties of Proposition 1.1 with respect to $\left(E_{n}\right)$. Because $G$ is ergodic, this observation proves

THEOREM 1.4. The following are equivalent:

(1) There exists a $G$-invariant mean $\varphi$ on $L_{\infty}(X, \beta, p)$ with $\varphi \neq I$.

(2) There exist a measurable set $E$, with $p(E)>0$, and an asymptotically invariant net $\left(A_{\alpha}\right)$, with $A_{\alpha} \subset X \backslash E$ for all $\alpha$.

If $G$ is countable, then in addition (1) is equivalent to

(3) There exists an arbitrarily small asymptotically invariant sequence.

We see then that the question of the existence of certain invariant linear functionals is equivalent to the much more concrete problem of the existence of certain types of asymptotically invariant nets of measurable sets.

2. Some applications. In this section, we make some applications of $\S 1$; the applications of $\S 1$ which give the theorems in the introduction follow in $\S 3$. Assume that $(X, \beta, p)$ is nonatomic and that $G$ is an ergodic semigroup of measure-preserving transformations of $(X, \beta, p)$. Given $g \in G$, let $g^{*}: L_{\infty}(X, \beta, p) \rightarrow L_{\infty}(X, \beta, p)$ be the adjoint of $f \rightarrow_{g} f$ for $f \in L_{1}(X, \beta, p)$. We say a mean $\varphi$ on $L_{\infty}(X, \beta, p)$ is $G^{*}$-invariant if $\varphi\left({ }_{g^{*}} F\right)=\varphi(F)$ for all $g \in G$ and $F \in L_{\infty}(X, \beta, p)$. If $G$ is a group, this is the same as the definition of invariance in $\S 1$. The techniques of $\S 1$ now apply to study $G^{*}$-invariant means.

In del Junco and Rosenblatt [2], it was shown that if $G$ is a countable amenable semigroup then (3) of Theorem 1.4 holds. This gives

THEOREM 2.1. If $G$ is a countable amenable semigroup of measure-preserving transformations of a nonatomic $(X, \beta, p)$, then $I$ is not the unique $G^{*}$-invariant mean.

It is an open question whether the assumption that $G$ is countable can be dropped in Theorem 2.1. This problem shares the same difficulty (the cardinality of $G$ ) with this open problem: does an amenable group $G$ acting on an infinite set $X$ always have more than one $G$-invariant mean on $l_{\infty}(X)$ ? Also, even when $G$ is countable, the cardinality of the set of invariant means on $L_{\infty}(X, \beta, p)$ is not known. 
Another application of $\S 1$ is to pairs of independent transformations. Let $X=[0,1]$ with Lebesgue measure $p$. Two measure-preserving transformations $g_{1}, g_{2}: X \rightarrow X$ are independent if $p\left(g_{1}^{-1}\left(E_{1}\right) \cap g_{2}^{-1}\left(E_{2}\right)\right)=p\left(E_{1}\right) p\left(E_{2}\right)$ for all measurable sets $E_{1}$ and $E_{2}$. One simple way of obtaining such a pair is this. Let $\theta$ : $[0,1] \rightarrow[0,1]^{2}$ be any Borel measure-preserving isomorphism of $[0,1]$ in Lebesgue measure $p$ with $[0,1]^{2}$ in Lebesgue measure $p \times p$. Let $\pi_{1}$ and $\pi_{2}$ be the first and second coordinate projections of $[0,1]^{2}$ onto $[0,1]$; and define $g_{i}=\pi_{i} \circ \theta$ for $i=1,2$. Then $g_{1}$ and $g_{2}$ are independent.

TheOREM 2.2. If $g_{1}, g_{2}:[0,1] \rightarrow[0,1]$ are independent measure-preserving transformations, then $I$ is the unique mean on $L_{\infty}([0,1], p)$ which is invariant under $g_{1}^{*}$ and $g_{2}^{*}$.

Proof. Suppose $I$ is not unique. Then there exists a sequence of measurable sets $\left(A_{n}\right)$ with $\lim _{n \rightarrow \infty} p\left(A_{n}\right)=0$ and

$$
\lim _{n \rightarrow \infty} p\left(g_{i}^{-1}\left(A_{n}\right) \cap A_{n}\right) / p\left(A_{n}\right)=1 \text { for } i=1,2 \text {. }
$$

But then we have

$$
\lim _{n \rightarrow \infty} p\left(g_{1}^{-1}\left(A_{n}\right) \cap g_{2}^{-1}\left(A_{n}\right)\right) / p\left(A_{n}\right)=1
$$

too. However, $p\left(g_{1}^{-1}\left(A_{n}\right) \cap g_{2}^{-1}\left(A_{n}\right)\right)=p\left(A_{n}\right)^{2}$. Hence, $0=\lim _{n \rightarrow \infty} p\left(A_{n}\right)=1$, a contradiction.

3. Permutations of the characters. In this section, we further specialize the theorems of $\S 1$ with the application to the group of automorphisms of a compact abelian group in mind. Let $G$ and $(X, \beta, p)$ be as in $\S 1$.

LeMma 3.1. Assume that a nontrivial asymptotically invariant net $\left(A_{\alpha}\right)$ exists for the action of $G$ on $X$. Then there exists a net $\left(F_{\alpha}\right) \subset L_{2}(X, \beta, p)$ such that

$$
\lim _{\alpha}\left\|_{g} F_{\alpha}-F_{\alpha}\right\|_{2}=0 \text { for all } g \in G,
$$

and $\left\|F_{\alpha}\right\|_{2}=1, \int F_{\alpha} d p=0$ for all $\alpha$.

Proof. Let $f_{\alpha}=\left(1_{A_{\alpha}} / \sqrt{p\left(A_{\alpha}\right)}\right)-\sqrt{p\left(A_{\alpha}\right)}$, and let $F_{\alpha}=f_{\alpha} /\left\|f_{\alpha}\right\|_{2}$ for all $\alpha$. Then $\int F_{\alpha} d p=0$ because $\int f_{\alpha} d p=0$, and $\left\|F_{\alpha}\right\|_{2}=1$ for all $\alpha$. Also,

$$
\left\|f_{\alpha}-f_{\alpha}\right\|_{2}^{2}=2\left(1-p\left(g^{-1} A_{\alpha} \cap A_{\alpha}\right) / p\left(A_{\alpha}\right)\right) \text {. }
$$

Since $\left\|f_{\alpha}\right\|_{2}^{2}=p\left(X \backslash A_{\alpha}\right)$ is bounded away from 0 by the nontriviality of $\left(A_{\alpha}\right)$, the sequence $\left(F_{\alpha}\right)$ has the desired invariance property by the asymptotic invariance of $\left(A_{\alpha}\right)$.

In Schmidt [14], asymptotically invariant nets with $p\left(A_{\alpha}\right)$ bounded away from 0 and 1 were studied. For the action of $S L(2, Z)$ on $T^{2}$, the existence of a sequence $\left(F_{n}\right)$ as in Lemma 3.1 was shown to be impossible via a combinatorial argument. We present here a more abstract argument which proves this same thing in particular and which will more directly relate Schmidt's idea to the nonamenability of $G$. It will also allow us to prove this same type of theorem for other groups of automorphisms of compact abelian groups. 
First, consider the case that $X$ is the $n$-dimensional torus $T^{n}, n \geqslant 2$, and $p$ is the normalized Haar measure on $T^{n}$. We coordinatize $T^{n}$ in the usual manner as $\left\{\left(z_{1}, \ldots, z_{n}\right): z_{i} \in C,\left|z_{i}\right|=1\right.$, for $\left.i=1, \ldots, n\right\}$. Let $G$ be the group of topological automorphisms of the compact group $T^{n}$. Then $G$ is a group of measure-preserving transformations of $T^{n}$; and each $g \in G$ has a unique representation as an $n \times n$ matrix $g=\left(g_{i j}\right)$ with integer entries and $\operatorname{det}\left(g_{i j}\right)= \pm 1$, where $g\left(z_{1}, \ldots, z_{n}\right)=$

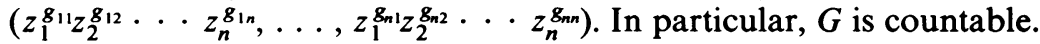

The space $L_{2}\left(T^{n}\right)$ has an orthonormal basis given by the characters $\Gamma\left(T^{n}\right)$. For each $\gamma \in \Gamma\left(T^{n}\right)$, there exists a uniquely determined sequence $\left(a_{1}, \ldots, a_{n}\right) \in Z^{n}$ such that $\gamma\left(z_{1}, \ldots, z_{n}\right)=z_{1}^{a_{1}} z_{2}^{a_{2}} \cdots z_{n}^{a_{n}}$. With this identification of $\Gamma\left(T^{n}\right)$, if $g \in G$ corresponds as before to $\left(g_{i j}\right)$, then

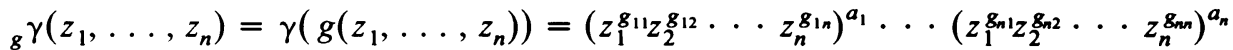

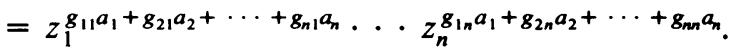

This gives the formula for ${ }_{g} \gamma$ which we did expect to be another character in $\Gamma\left(T^{n}\right)$; it is the character which corresponds to the image $\left(b_{1}, \ldots, b_{n}\right)=$ $\left(g_{i j}\right)^{T}\left(a_{1}, \ldots, a_{n}\right)$ of $\left(a_{1}, \ldots, a_{n}\right)$ under the transposed matrix $\left(g_{i j}\right)^{T}$. Hence, up to this identification of $\Gamma\left(T^{n}\right)$ with $Z^{n}$, the regular action of $G$ on $L_{2}\left(T^{n}\right)$ permutes $\Gamma\left(T^{n}\right)$ exactly as the transposes of the matrices $\left(g_{i j}\right)$ act on $Z^{n}$. Therefore, if we let $\theta: L_{2}\left(T^{n}\right) \rightarrow l_{2}\left(Z^{n}\right)$ be the canonical identification via the Riesz-Fischer theorem using the above identification of $\Gamma\left(T^{n}\right)$ and $Z^{n}$, then $\theta\left({ }_{g} F\right)(v)=\theta(F)\left(\left(\left(g_{i j}\right)^{T}\right)^{-1} v\right)$ for each $g \in G$ and its corresponding matrix $\left(g_{i j}\right)$, each $F \in L_{2}\left(T^{n}\right)$, and $v \in Z^{n}$. Notice that $\left(\left(g_{i j}\right)^{T}\right)^{-1}$ is again a matrix with integer entries and determinant \pm 1 . Since it should not cause confusion, we will also think of $G$ as a group of invertible matrices acting on $Z^{n}$.

More generally, suppose $X$ is a compact abelian group and $G$ is the group of topological automorphisms of $X$. Then $G$ acts as measure-preserving transformations of $\left(X, \beta, \lambda_{X}\right)$. The character group $\Gamma$ of $X$ forms an orthonormal basis of $L_{2}\left(X, \beta, \lambda_{X}\right)$ and the regular action of $G$ on $L_{2}\left(X, \beta, \lambda_{X}\right)$ permutes $\Gamma$. Let $\theta$ : $L_{2}\left(X, \beta, \lambda_{X}\right) \rightarrow l_{2}(\Gamma)$ be the canonical identification via the Riesz-Fischer theorem. The group $G$ acts on $l_{2}(\Gamma)$ by ${ }_{g} f(\gamma)=f(\gamma \circ g)$ for all $g \in G, f \in l_{2}(\Gamma)$, and $\gamma \in \Gamma$. With this action $\theta\left({ }_{g} F\right)={ }_{g^{-1}} \theta(F)$ for all $g \in G, F \in L_{2}\left(X, \beta, \lambda_{X}\right)$.

The group $G$ above permutes $\Gamma \backslash\{1\}$ because 1 is fixed by any $g \in G$. We can speak of $G$-invariant means on $l_{\infty}(\Gamma \backslash\{1\})$ in the usual way with invariance with respect to the regular action of $G$ on $l_{\infty}(\Gamma \backslash\{1\})$. The comments above give this proposition and its immediate corollary for $T^{n}, n \geqslant 2$.

PROPOSITION 3.2. If there is a nontrivial asymptotically invariant net for the group $G$ of topological automorphisms of the compact abelian group $X$, then there exists $a$ $G$-invariant mean on $l_{\infty}(\Gamma \backslash\{1\})$.

Proof. Let $\left(A_{\alpha}\right)$ be the asymptotically invariant net. By Lemma 3.1 and the comments above, there exists a net $\left(f_{\alpha}\right) \subset l_{2}(\Gamma \backslash\{1\})$ such that $\lim _{\alpha}\left\|_{g} f_{\alpha}-f_{\alpha}\right\|_{2}=0$ for all $g \in G$ and $\left\|f_{\alpha}\right\|_{2}=1$ for all $\alpha$. Let $h_{\alpha}=\left|f_{\alpha}\right|^{2}$ for all $\alpha$. Then for all $g \in G$,

$$
\begin{aligned}
\left\|h_{g} h_{\alpha}-h_{\alpha}\right\|_{1} & =\left\|\left({ }_{g}\left|f_{\alpha}\right|-\left|f_{\alpha}\right|\right)\left({ }_{g}\left|f_{\alpha}\right|+\left|f_{\alpha}\right|\right)\right\|_{1} \\
& \leqslant\left\|_{g}\left|f_{\alpha}\right|-\left|f_{\alpha}\right|\right\|_{2}\left\|_{8}\left|f_{\alpha}\right|+\left|f_{\alpha}\right|\right\|_{2} \leqslant 2\left\|_{g} f_{\alpha}-f_{\alpha}\right\|_{2} .
\end{aligned}
$$


Hence, $\lim _{\alpha}\left\|_{g} h_{\alpha}-h_{\alpha}\right\|_{1}=0$ for all $g \in G$. Because $\left(h_{\alpha}\right) \subset l_{1}(\Gamma \backslash\{1\})$, any $\omega^{*}$-limit of $\left(h_{\alpha}\right)$ in $l_{\infty}^{*}(\Gamma \backslash\{1\})$ is a $G$-invariant mean on $l_{\infty}(\Gamma \backslash\{1\})$.

REMARK. The group $G$ can be replaced by any subgroup $G$ in this proposition.

COROLLARY 3.3. If there were a nontrivial asymptotically invariant sequence for the group $G$ of topological automorphisms of $T^{n}, n \geqslant 2$, then there would be a G-invariant mean on $l_{\infty}\left(Z^{n} \backslash\{0\}\right)$.

Again, consider the case that $X=T^{n}, n \geqslant 2$. Because $G$ as a matrix group contains $S L(n, Z)$, it contains free nonabelian subgroups and it is not amenable. If one has a free action of $G$ on a set $Y$, then the existence of a $G$-invariant mean on $l_{\infty}(Y)$ would imply that $G$ is amenable, an impossibility. So Corollary 3.3 is close to giving Theorem 3.4 except that the action of $G$ on $Z^{n} \backslash\{0\}$ is not a free action. The same difficulty occurs in the general case of a compact abelian group $X$; in order to use Proposition 3.2 to assert there cannot be nontrivial asymptotically $G$-invariant nets in $X$, we would need besides the nonamenability of $G$, some information about the orbit structure of the action of $G$ on $\Gamma \backslash\{1\}$. This is the purpose of Proposition 3.5.

THEOREM 3.4. The Haar integral is the unique mean on $L_{\infty}\left(T^{n}, \beta, \lambda_{T^{n}}\right), n \geqslant 2$, invariant under the topological automorphisms of $T^{n}$.

Proof. The results in $\S 1$ and Corollary 3.3 show that we need to prove there is no $S L(n, Z)$-invariant mean on $l_{\infty}\left(Z^{n} \backslash\{0\}\right)$. For $n=2$, this was stated in Mycielski [7]; here is a proof. A $2 \times 2$ integer matrix $\left(\begin{array}{ll}a & b \\ c & d\end{array}\right)$ with $a d-b c=1$ and $a+d=2$ is called parabolic. It is easy to check that $\left(\begin{array}{ll}a & b \\ c & d\end{array}\right)$ is parabolic in $\operatorname{SL}(2, Z)$ if and only if 1 is an eigenvalue. W. Magnus and B. H. Neumann have proved (see [7, p. 725]) that there exist free nonabelian subgroups of $S l(2, Z)$ without any parabolic elements except the identity matrix. Let $F \subseteq S L(2, Z)$ be such a free group. Then $F$ acts freely on $Z^{2} \backslash\{0\}$ and $F$ is nonamenable, so there cannot exist an $F$-invariant mean on $l_{\infty}(Z \backslash\{0\})$.

For $n>2$, we argue by induction. Suppose that we have shown that $l_{\infty}\left(Z^{n} \backslash\{0\}\right)$ has no $S L(n, Z)$-invariant mean. Then by Tarski's theorem (see [9]), $Z^{k} \backslash\{0\}$ has paradoxical decompositions relative to $S L(n, Z)$. Hence, $Z \times$ $\left(Z^{n} \backslash\{0\}\right)=\left\{\left(z_{1}, \ldots, z_{n+1}\right)\right.$ : some $\left.z_{i} \neq 0, i=2, \ldots, n+1\right\}$ has paradoxical decompositions relative to $S L(n+1, Z)$. Let $g\left(z_{1}, z_{2}, \ldots, z_{n+1}\right)=\left(z_{1}, z_{2}, \ldots, z_{n+1}\right.$ $\left.+z_{1}\right)$ for $\left(z_{1}, \ldots, z_{n+1}\right) \in Z^{n+1}$; then $g \in S L(n+1, Z)$. Also, $g$ maps $\left(Z^{n+1} \backslash\{0\}\right) \backslash\left(Z \times\left(Z^{n} \backslash\{0\}\right)\right)$ into $Z \times\left(Z^{n} \backslash\{0\}\right)$; hence $Z^{n+1} \backslash\{0\}$ has paradoxical decompositions relative to $S L(n+1, Z)$ (see Lemma 1 in [9]). Thus, there is no $S L(n+1, Z)$-invariant mean on $l_{\infty}\left(Z^{n+1} \backslash\{0\}\right)$.

For general compact abelian groups we need this proposition.

Proposition 3.5. Suppose $G$ is a group acting on a set $Y$ and suppose there exists $\left\{y_{\alpha}: \alpha \in A\right\} \subset Y$ such that $\left\{G y_{\alpha}: \alpha \in A\right\}$ is a partition of $Y$. Let $H_{\alpha}=\{g \in G$ : $\left.g y_{\alpha}=y_{\alpha}\right\}$ for $\alpha \in A$ and suppose $H_{\alpha}$ is amenable for all $\alpha \in A$. Then if there exists $a$ $G$-invariant mean on $l_{\infty}(X)$, the group $G$ itself is amenable. 
Proof. Let $\varphi_{\alpha}$ be an $H_{\alpha}$-invariant mean on $l_{\infty}\left(H_{\alpha}\right)$ and let $\Phi$ be a $G$-invariant mean on $l_{\infty}(Y)$. We identify the orbit $G y_{\alpha}$ with $G / H_{\alpha}$ by the correspondence $g H_{\alpha} \leftrightarrow g y_{\alpha}$ for $g \in G$. Because this commutes with the usual action of $G$ on the left-coset spaces $G / H_{\alpha}$, there is no harm in assuming at the outset that $Y=$ $\cup\left\{Y_{\alpha}: \alpha \in A\right\}$, a partition of $Y$, each $Y_{\alpha}=G / H_{\alpha}$, and $G$ acts on $Y$ by left-multiplication in each $Y_{\alpha}$. Fix $F \in l_{\infty}(G)$ and define $F\left(\varphi_{\alpha}\right) \in l_{\infty}\left(Y_{\alpha}\right)$ by $F\left(\varphi_{\alpha}\right)\left(g H_{\alpha}\right)=\varphi_{\alpha}(F(g \cdot))$ where $F(g \cdot)$ is the function in $l_{\infty}\left(H_{\alpha}\right)$ given by $F(g \cdot)(h)=F(g h)$ for all $h \in H_{\alpha}$. This is a well-defined element $F\left(\varphi_{\alpha}\right) \in$ $l_{\infty}\left(G / H_{\alpha}\right)$ because $\varphi_{\alpha}$ is $H_{\alpha}$-invariant. Now define $F_{Y}\left(g H_{\alpha}\right)=F\left(\varphi_{\alpha}\right)\left(g H_{\alpha}\right)$ for all $g \in G$ and $\alpha \in A$. This is well defined because $Y$ is partitioned by the coset spaces and it gives $F_{Y} \in l_{\infty}(Y)$. We get a linear functional $\psi$ on $l_{\infty}(G)$ by $\psi(F)=\Phi\left(F_{Y}\right)$ for all $F \in l_{\infty}(G)$. It is easy to check that $\psi$ is a mean on $l_{\infty}(G)$. So to prove $G$ is amenable only requires showing $\psi$ is $G$-invariant. But for $g_{0} \in G$ and $F \in l_{\infty}(G)$, $\left({ }_{g_{0}} F\right)\left(\varphi_{\alpha}\right)\left(g H_{\alpha}\right)=F\left(\varphi_{\alpha}\right)\left(g_{0} g H_{\alpha}\right)$ for all $g \in G$ and $\alpha \in A$. Therefore, $\left({ }_{g_{0}} F\right)_{Y}$ $={ }_{g_{0}}\left(F_{Y}\right)$; so $\left.\psi\left(g_{g_{0}} F\right)=\Phi\left({\left(g_{0}\right.}_{F}\right)_{Y}\right)=\Phi\left({ }_{g_{0}}\left(F_{Y}\right)\right)=\Phi\left(F_{Y}\right)=\psi(F)$ for all $g_{0} \in G$ and $F \in l_{\infty}(G)$.

Remark. When $G$ is transitive on $Y$ and $H=\left\{g \in G: g x_{0}=x_{0}\right\}$ is normal in $G$ for some fixed $x_{0}$, this theorem just states the well-known fact that when $H$ and $G / H$ are amenable, then $G$ is amenable. For applications, it is often important that the subgroups $H_{\alpha}$ are not assumed to be normal.

One application of Proposition 3.5 is that it gives another proof of Theorem 3.4. The case $n \geqslant 3$ is derived by induction, as in the proof of Theorem 3.4, from the case $n=2$. For $n=2$, we know $G$ is not amenable because $G \supset S L(2, Z)$ which contains free nonabelian subgroups. Let $x_{n}=(n, 0) \in Z \backslash\{0\}$. Then $D_{n}=G x_{n}=$ $\{(n p, n q): p, q \in Z$ and $\operatorname{gcd}(p, q)=1\}$ describes the orbit of $x_{n}$ under $G$. Also, because $\left(D_{n}: n \in Z \backslash\{0\}\right)$ is a partition of $Z^{2} \backslash\{0\}$, this gives the orbit structure of $G$ acting on $Z^{2} \backslash\{0\}$. Let $H_{n} \subset G$ be the subgroup consisting of all $g \in G$ such that $g x_{n}=x_{n}$. Then for all $n \in Z \backslash\{0\}, H_{n}$ is the same subgroup, $\left\{\left(\begin{array}{cc}1 & a \\ 0 & \pm 1\end{array}\right): a \in Z\right\}$. This has an abelian normal subgroup $\left\{\left(\begin{array}{cc}1 & a \\ 0 & 1\end{array}\right): a \in Z\right\}$ of index 2; therefore, $H_{n}$ is the same amenable subgroup of $G$ for all $n \in Z \backslash\{0\}$. By Proposition 3.5, there does not exist a $G$-invariant mean on $l_{\infty}\left(Z^{2} \backslash\{0\}\right)$.

Another application of Proposition 3.5 is that it gives this general theorem which essentially appears in Losert and Rinder [5].

THEOREM 3.6. Let $G_{0}$ be a subgroup of the group of topological automorphisms of $X$ which acts ergodically on $\left(X, \beta, \lambda_{X}\right)$. Assume $G_{0}$ is not amenable and that there exist characters $\left\{\gamma_{\alpha}: \alpha \in A\right\} \subset \Gamma \backslash\{1\}$ such that each $H_{\alpha}=\left\{g \in G_{0}: \gamma_{\alpha} \circ g=\gamma_{\alpha}\right\}$ is amenable, and $\left\{g \gamma_{\alpha}: g \in G_{0}, \alpha \in A\right\}=\Gamma \backslash\{1\}$. Then the Haar integral is the unique $G_{0}$-invariant mean on $L_{\infty}\left(X, \beta, \lambda_{X}\right)$.

Proof. If the Haar integral were not unique, then the ergodicity of $G_{0}$ and Theorem 1.3 would prove there exists an arbitrarily small asymptotically invariant net $\left(A_{\alpha}\right)$ for the action of $G_{0}$ on $X$. By Proposition 3.2, there would be a $G_{0}$-invariant mean on $l_{\infty}(\Gamma \backslash\{1\})$. This is impossible by Proposition 3.5.

Here are some other examples of compact abelian groups with unique means. 
THEOREM 3.7. Let $K$ be a compact abelian group with at least two elements and let $X=\Pi_{n=1}^{\infty} K$ be the product compact abelian group. Then the topological automorphisms $G$ of $X$ have the Haar integral $I$ as the unique $G$-invariant mean $L_{\infty}\left(X, \beta, \lambda_{X}\right)$.

THEOREM 3.8. Let $\mu$ be any probability measure on $\{0,1\}$ with $\mu\{0\}$ and $\mu\{1\}$ both not zero. Let $X=\prod_{n=1}^{\infty}\{0,1\}$ with the product probability measure $p=\prod_{n=1}^{\infty} \mu$. The permutations $G$ of $Z^{+}$act as measure-preserving transformations of $X$ by permuting the coordinates; this group $G$ has the integral $I$ as the unique $G$-invariant mean on $L_{\infty}(X, \beta, p)$.

Proof. The proofs for the two theorems are similar; we will prove Theorem 3.8 with $\mu(\{0\})=\mu(\{1\})=1 / 2$. In this case $G$ again consists of topological automorphisms of $X$ considered as a compact group by identifying $\{0,1\}$ with $Z / 2 Z$. So one needs only prove that the character group $\Gamma$ of $X$ is acted on by $G$ in such a manner that $l_{\infty}(\Gamma \backslash\{1\})$ admits no $G$-invariant mean. But this character group $\Gamma=\bigcup_{k=0}^{\infty} \Gamma_{k}$ where $\Gamma_{0}=\{1\}$ and $\Gamma_{k}, k \geqslant 1$, consists of those characters which depend on exactly $k$ coordinates. The group $G$ leaves each $\Gamma_{k}$ invariant and $\left\{\Gamma_{k}\right.$ : $k \geqslant 0\}$ forms a partition of $\Gamma$. Because of Proposition 3.5, it would suffice to find two elements $g_{1}, g_{2} \in G$ which are free group generators and such that the group $H$ generated by $\left\{g_{1}, g_{2}\right\}$ acts freely on each $\Gamma_{k}, k \geqslant 1$. This is easily, but not explicitly, done by identifying the coordinate set $Z^{+}$with the free group $F_{2}$ on free generators $a_{1}$ and $a_{2}$, and letting $g_{i}$ be the permutation of $Z^{+}$corresponding to left-multiplication of $a_{i}$ on $F_{2}, i=1,2$. Then if $h \in H$ and $\gamma \in \Gamma_{k}, k \geqslant 1$, satisfy $h \gamma=\gamma$, one knows that the finite set $F \subset Z^{+}$, with $k$ elements, that consists of the coordinates in which $\gamma$ is nontrivial must satisfy $h F=F$. It follows that there exist $N \geqslant 1$ and $z \in F$ with $h^{N} z=z$; hence, $h^{N}=e$ and $h=e$ as a group element. Thus, the action by this group $H$ on $\Gamma_{k}$ is free.

It is shown in Trott [17] that the group $G$ of topological automorphisms of $T^{n}$, $n \geqslant 2$, is generated by the two elements $g_{1}, g_{2}$ given by $g_{1}\left(z_{1}, \ldots, z_{n}\right)=$ $\left(z_{1}, z_{1} z_{2}, z_{3}, \ldots, z_{n}\right)$ and $g_{2}\left(z_{1}, \ldots, z_{n}\right)=\left(z_{2}, \ldots, z_{n}, z_{1}^{(-1)^{n}}\right)$ for $\left(z_{1}, \ldots, z_{n}\right) \in T^{n}$. Because $g_{2}$ has finite order, an equivalent statement of Theorem 3.4 is that there cannot be a nontrivial sequence $\left(A_{n}\right)$ with $g_{2}\left(A_{n}\right)=A_{n}$ for all $n \geqslant 1$, which is also asymptotically invariant under $g_{1}$. Applying this to cylinder sets $A \times A \subset T^{2}$ gives this interesting corollary.

Corollary 3.9. For $\delta, 0<\delta<1$, there exists $\varepsilon>0$ such that for all measurable sets $A \subset T^{1}$ with Haar measure $p(A) \leqslant \delta$,

$$
\int_{A} p(A z \cap A) d p(z) \leqslant(1-\varepsilon) p(A)^{2} .
$$

It seems to be difficult to give an elementary proof of this fact. For this reason, it is also difficult to find the largest $\varepsilon=\varepsilon(\delta)$ for a given $\delta>0$. For example, calculations with various cases suggest that if $\delta=1 / 2$, then $\varepsilon=1 / 4$ will work, but this has not been proven except when $p(A)=\delta$ too.

We now prove the two theorems discussed in the introduction. 
Proof of Theorem 3.10. Suppose $f$ satisfies (a), (b), and $\left(\mathrm{c}_{0}\right)$. We identify $J^{n}$ with $T^{n}$ in the usual way using the exponential map exp: $J^{n} \rightarrow T^{n}, \exp \left(x_{1}, \ldots, x_{n}\right)$ $=\left(\exp \left(2 \pi i x_{1}\right), \ldots, \exp \left(2 \pi i x_{n}\right)\right)$ for all $\left(x_{1}, \ldots, x_{n}\right) \in J^{n}$. Because $f$ is absolutely continuous with respect to Lebesgue measure, by restricting $f$ to $J^{n}, J=[0,1]$, we can define a mean $\varphi$ on $L_{\infty}\left(T^{n}, \beta, \lambda_{T^{n}}\right)$ with $\varphi\left(1_{\exp (E)}\right)=f(E)$ for all measurable sets $E \subset J^{n}$. Since $f$ is invariant by isometries and $\left(x_{1}, \ldots, x_{n}\right) \mapsto\left(x_{1}+\right.$ $\left.x_{2}, x_{2}, \ldots, x_{n}\right)$, Trott [17] shows that $\varphi$ is invariant under topological automorphisms of $T^{n}$. By Theorem 3.4, $\varphi$ is the Haar integral. But then $F(E)=\lambda_{n}(E)$ for measurable $E \subset J^{n}$; by translation invariance of $f, f(E)=\lambda_{n}(E)$ for all bounded measurable sets $E$.

Proof of Theorem 3.11. First, note that invariance under the first two transformations implies that $f$ is invariant under translations by $Z^{n}$. Then use Trott [17] to show that at least the last two transformations generate a subgroup $G$ of finite index in $S L(n, Z)$. Indeed, Trott claims that if $n$ is odd, $G=S L(n, Z)$; if $n=2$, one can check easily that $G=S L(n, Z)$. Otherwise, it is not hard to prove from Trott [17] that the left cosets of $G$ in $S L(n, Z)$ are among $\{\sigma G: \sigma \in S L(n, Z), \sigma$ diagonal $\}$. Now if the mean $\varphi$ above is not the Haar integral, then $l_{\infty}\left(Z^{n} \backslash\{0\}\right)$ admits a $G$-invariant mean. But because $G$ is finite index in $S L(n, Z)$, it follows that $l_{\infty}\left(Z^{n} \backslash\{0\}\right)$ admits an $S L(n, Z)$-invariant mean. Since this is impossible, $\varphi$ is the Haar integral and $f$ is the Lebesgue measure.

Remark. For $R^{2}$ the conditions (a), (b), ( $\left.\mathrm{c}_{0}\right)$, and the absolute continuity of $f$, without the additional assumption of Theorem 3.11 do not suffice for the conclusion of Theorem 3.11. This follows for instance from Theorem 6.1 [8] and the observation that for $R^{n}, n=1,2$, or $S^{n}, n=1$, there exists an ideal 9 of measurable subsets of $\beta$ which is invariant under isometries, contains all null sets, contains a set of positive measure (e.g. some nowhere dense set), but does not contain $J^{n}$ or $S^{n}$ respectively.

There are some more facts to observe about the role of null sets in Theorems 3.10 and 3.11. Consider the Haar measure $\lambda=\lambda_{T^{2}}$ on Lebesgue measurable subsets of $T^{2}$; let $G$ be the topological automorphisms of $T^{2}$. There do exist $G$-invariant finitely-additive positive measures $\mu$ on $\beta$ with $\mu\left(T^{2}\right)=1$ but with $\mu \neq \lambda$. For example, let $W_{M}=\left\{z \in T^{2}: z\right.$ has finite order dividing $\left.M !\right\}$; then $W_{M}$ is a finite subgroup of $T^{2}$ which is permuted by $G$. By taking $\omega^{*}$-limits of counting measures on $W_{M}$, one can construct a $G$-invariant finitely-additive positive measure $\mu\left(\cup_{M=1}^{\infty} W_{M}\right)=\mu\left(T^{2}\right)=1$. Of course, $\lambda\left(\cup_{M=1}^{\infty} W_{M}\right)=0$. We can arrange having $\mu$ vanish on finite sets here. These two problems are unsolved: (1) if $\mu$ is a $G$-invariant finitely-additive positive measure on $\beta$ with $\mu\left(T^{2}\right)=1$ and $\mu\left(\cup_{M=1}^{\infty} W_{M}\right)=0$, is $\mu=\lambda$ ?, (2) if $\mu$ is as in (1) and $\mu(N)=0$ for all $N$ countably infinite, is $\mu=\lambda$ ?

We can also formulate Theorem 3.4 with a weaker assumption than the implied absolute continuity. Let $G$ and $\left(T^{n}, \beta, \lambda_{T^{n}}\right)=\left(T^{n}, \beta, \lambda_{n}\right)$ be as in Theorem 3.4. For each $g \in G$, let $F_{g}=\left\{z \in T^{n}: g z=z\right\}$. Then $F_{g}$ is a closed subgroup and $\lambda_{n}\left(F_{g}\right)=0$ if $g$ is not the identity $e$. Let $F=\cup\left\{F_{g}: g \in G \backslash\{e\}\right\}$. Then $\lambda_{n}(F)=$ 0 too. 
THEOREM 3.12. Let $\mu$ be a $G$-invariant finitely-additive positive probability measure on $\left(T^{n}, \beta\right)$ with $\mu(F)=0$. Then $\mu=\lambda_{n}$.

Proof. It is sufficient to prove $\mu(N)=0$ for all null sets $N$. If $\mu(N)>0$ with $\lambda(N)=0$, then let $X=\cup\{g N: g \in G\} \backslash F$. Since $F$ is $G$-invariant and $G$ is countable, $X$ is a $G$-invariant null set with $\mu(X)>0$. Define $\nu(E)=\mu(E) / \mu(X)$ for all $E \subset X$ (any $E \subset X$ is measurable). This gives a $G$-invariant finitely-additive probability measure on $X$. But $G$ is not amenable and $G$ acts freely on $X$, a contradiction.

We end with two questions. First, we would like to know if the amenability of $G$ has anything to do with the uniqueness of $I$. For example, is it the case that $I$ fails to be unique if and only if $X=X_{1} \times X_{2}$ as a nontrivial product of two probability spaces, $G=G_{1} \times G_{2}$ with $G_{i}$ acting on $X_{i}$ as a group of measure-preserving transformations for $i=1,2, G$ acts on $X$ by $\left(g_{1}, g_{2}\right)\left(x_{1}, x_{2}\right)=\left(g_{1} x_{1}, g_{2} x_{2}\right)$ for all $g_{i} \in G_{i}$ and $x_{i} \in X_{i}$ for $i=1,2$, and $G_{1}$ is amenable?

The theorems presented here suggest that the Lebesgue integral $I$ might indeed be the unique isometry invariant mean on $L_{\infty}\left(S^{2}\right)$. Using the technique of $\S 1$ and $\S 2$, this question becomes a problem in representation theory for connected nonabelian compact groups. Let $G$ be any connected compact Lie group with no $T^{k}, k \geqslant 1$, as a closed normal subgroup. Let $\left(U_{k}: k>1\right)$ be a list up to a unitary equivalence of the irreducible representations of $G$ on finite-dimensional Hilbert spaces $\left(H_{k}: k \geqslant 1\right)$. Exclude the trivial representation from this list. Under what conditions (if ever!) is it the case that for each finite set $F \subset G$, there exists a sequence $\left(x_{k}\right), x_{k} \in H_{k},\left\|x_{k}\right\|_{H_{k}}=1$ for all $k \geqslant 1$, such that $\lim _{k \rightarrow \infty}\left\|U_{g}^{k} x_{k}-x_{k}\right\|_{H_{k}}$ $=0$ for all $g \in F$ ? If $G$ is a group for which this fails for some finite $F$, then $L_{\infty}\left(G, \beta, \lambda_{G}\right)$ has the Haar integral as the unique $G$-invariant mean. If this condition holds then the regular representation of $G$ as a discrete group is weakly contained in the orthogonal complement of $\{1\}$ in $L_{2}\left(G, \beta, \lambda_{G}\right)$ and, also, there will be a nontrivial asymptotically invariant net for the left-multiplication of $G$ on $G$. This problem on the irreducible representations of $G$ is fundamental for the solution of the problem of Ruziewicz which Banach studied in [1].

Postscript. There have been some new results related to the above which are worth mentioning. K. Schmidt [15] has shown that for a countable ergodic group $G$ of measure-preserving transformations of $(X, \beta, p)$, there is more than one $G$-invariant mean on $L_{\infty}(X, \beta, p)$ if and only if the identity representation is weakly contained in the regular representation of $G$ as a discrete group when it is restricted to $L_{2}^{0}(X, \beta, p)=\left\{f \in L_{2}(X, \beta, p): \int f d p=0\right\}$. He also discussed the relationships of strong ergodicity, Kazhdan's property $T$, and the uniqueness of $G$-invariant means. His remarks on Kazhdan's property $T$ make it clear that one can embed $S L(3, Z)$ as a dense subgroup of a totally disconnected compact metric group $G$ and thus construct a compact group $G$ for which the Haar integral is the unique $G$-invariant mean on $L_{\infty}\left(G, \beta, \lambda_{G}\right)$.

Also, using some of the ideas of this paper and Kazhdan's property $T$, Dennis Sullivan [16] has solved the Banach-Ruziewicz problem for $n>5$; that is, he has 
shown that for $n \geqslant 5$, the additional mapping is not needed in Theorem 3.10. He shows that for $n \geqslant 5$, the orthogonal group $O_{n}$ contains a dense subgroup $\Gamma_{n}$ which as a discrete group has Kazhdan's property $T$ and, hence, the compact group $O_{n}$ has the Haar integral as the unique $O_{n}$-invariant mean on $L_{\infty}\left(O_{n}, \beta, \lambda_{O_{n}}\right)$.

\section{REFERENCES}

1. S. Banach, Sur le probleme de la mesure, Oeuvres, Vol. I, PWN, Warsaw, 1967, pp. 318-322.

2. A. del Junco and J. Rosenblatt, Counterexamples in ergodic theory and number theory, Math. Ann. 245 (1979), 185-197.

3. E. Granirer, Criteria for compactness and for discreteness of locally compact amenable groups, Proc. Amer. Math. Soc. 40 (1973), 615-624.

4. F. P. Greenleaf, Invariant means on topological groups and their applications, Van Nostrand, New York, 1969.

5. V. Losert and H. Rindler, Almost invariant sets (preprint).

6. E. Marczewski (Szprilrajn), Problem 169, The Scottish Book, 1937-1938.

7. J. Mycielski, Equations insoluable on $G L_{2}(C)$ and related problems, Amer. Math. Monthly 84 (1977), 723-726; 85 (1978), 263-265.

8. __ Finitely-additive invariant measures. I, Colloq. Math. 42 (1979), 309-318.

9. , Finitely-additive invariant measures. III, Colloq. Math. (to appear).

10. I. Namioka, Folner's conditions for amenable semigroups, Math. Scand. 15 (1964), 18-28.

11. J. Rosenblatt, Finitely-additive invariant measures. II, Colloq. Math. 42 (1979), 361-363.

12. Invariant means for the bounded measurable functions on a non-discrete locally compact group, Math. Ann. 220 (1976), 219-228.

13. W. Rudin, Invariant means on $L_{\infty}$, Studia Math. 44 (1972), 219-227.

14. K. Schmidt, Asymptotically invariant sequences and an action of $S L(2, Z)$ on the 2-sphere (preprint).

15. Amenability, Kazhdan's property $T$, strong ergodicity, and invariant means for ergodic actions (preprint).

16. D. Sullivan, For $n>3$ there is only one finitely-additive rotationally invariant measure on the n-sphere defined on all Lebesgue measurable sets, Bull. Amer. Math. Soc. (N.S.) 1 (1981), 121-123.

17. S. Trott, A pair of generators for the unimodular group, Canad. Math. Bull. 5 (1962), 245-252.

Department of Mathematics, Ohio State University, Columbus, Ohoo 43210 